\title{
An efficient and secure data storage in cloud computing using modified RSA public key cryptosystem
}

\author{
Y. Kiran Kumar ${ }^{1}$, R. Mahammad Shafi ${ }^{2}$ \\ ${ }^{1}$ Department of Computer Science, Bharathiar University, Coimbatore, India \\ ${ }^{2}$ Bharathiar University, Coimbatore, India
}

\begin{tabular}{l}
\hline \hline Article Info \\
\hline Article history: \\
Received Apr 16, 2019 \\
Revised Aug 17, 2019 \\
Accepted Aug 29, 2019
\end{tabular}

\section{Keywords:}

Cloud data storage

Cloud service models

Data security

Modified RSA

Public key cryptosystem

RSA

\begin{abstract}
Cloud Computing is the ability to improve the utility or train new human resources without investing in new infrastructure, or add capabilities to existence without the latest software licensing. It expanded the capabilities of Information Technology (IT). From the past few years, cloud computing has developed from a good business concept in the best rising sectors of the IT industry. But more information on individuals and companies was put in the cloud, and concerns began to think about how secure the cloud environment was. Despite cloud surrounding structures, enterprise users still do not want to expand their business in the cloud. Security reduces the growth of cloud computing and continues to spread the market with complexity with data privacy and data protection. The security of cloud computing has constantly been an significant aspect of improved quality of service from cloud service providers. Data storage in the cloud has a problem related to data security. However, cloud computing construct many new security challenges which have not been well examine. In order to ensure that the user's data in the cloud is secure, we have proposed an effective mechanism with a distinctive feature of data integrity and privacy. This paper focusing on problems relating to the cloud data storage techniques and security in virtual environment. We recommend a method for providing data storage and security in cloud using public key Cryptosystem, which uses the concept of the modified RSA algorithm to provide better security for the data stored in the cloud.
\end{abstract}

Copyright () 2020 Institute of Advanced Engineering and Science. All rights reserved.

\section{Corresponding Author:}

R. Mahammad Shafi,

Research Spervisor, Bharathiar University,

Coimbatore, India,

Email: rmdshafi@gmail.com

\section{INTRODUCTION}

Cloud Computing has many unique features that make it very valuable. Different types of services can be easily accessible from cloud computing. Cloud Computing services provide client-specific applications and Data storage through dedicated cloud servers. That means there are no data and applications on the computer used by the client. Only the operating system and the cloud computing service will be the only application. The client will have to access the applications and data so that he can directly access from cloud server. This whole process is called cloud computing. Using cloud computing does not have the risk of software, crash, and data loss. Moreover, the client-utilized device also works faster. With virtualization technologies, cloud computing uses more efficient resources [1]. Cloud Computing services are growing and expected to expand in the future. The physical server runs many virtual machines and operating systems. Cloud Computing has a facility to use computing resources directly through the Internet. Computing source may be a combination of software or hardware, or both, and computing resources will be released in the form of Internet services. A service allows the user to access the computing 
resource. Services operate on a remote server and users access the interface as a browser. Cloud computing stores data stored on a user's remote cloud server [2]. Information technologies take new waves in order to change civilizations. Computing significantly expanded, making the database more robust.

Cloud computing detects briefly that cloud computing services offer client-specific applications and data storage through dedicated Web servers. In Cloud Computing all data is stored on a web server. There are no data and applications on the client-utilized computer [3]. Only the operating system and the cloud computing service will be the only application. Client Applications and data are accessible to get cloud servers directly from cloud servers. There is no risk of software, crash and databases using cloud computing, the client-utilized device also works faster.

\section{CLOUD COMPUTING DEPLOYMENT MODELS}

In Cloud Computing each model has a unique value proposition, which provides a line improvement provider for the business and provides customer service option. The following is some of the major or popular cloud operation models.

a. Private Cloud: Private Cloud infrastructure is controlled by a company or a third party and only maintains the requirements of the company.

b. Public Cloud: Public Cloud infrastructure is available to a large organizations or general public and owned by the vendor who provides cloud services.

c. Community Cloud: Community Cloud infrastructure is a shared community that has operated by one or more organizations and supports the same services.

d. Hybrid Cloud: Hybrid Cloud infrastructure is a combination of two or more different cloud infrastructures like private, public or community clouds, but portability of data and applications provided by standard technology.

\section{CLOUD COMPUTING SERVICES} basictypes:

The wide range of services offered by cloud computing organizations can be categorized into three

\subsection{Software as a service (SaaS)}

Many users allow access to the application when SaaS services provide better accessibility and active functionality. SaaS services provide focused business operations. The cloud resource administrator creates controls regarding users' access to applications.

\subsection{Platform as a service (PaaS)}

User configuration under PaaS does not use the applications and facilities provided by the cloud provider. Cloud storage options are available to use cloud needs. The model pre-runner manages all cloud computing designs. It provides support for applications and services and provides computational resources through the host platform.

\subsection{Infrastructure as a servive (IaaS)}

This environment can include hardware, operating systems, network connectivity and other IT resources. The IT resources provided by IaaS are generally not pre configured, placing the administrative responsibility directly upon the cloud consumer. The user of the PaaS dedicated API makes it possible to organize the activities of the server hosting engine to run and reproduce the environment based on user requests. Each provider is based on its API related key capabilities, the app developed for each particular group provider is not moved to another cloud host, but its API is excluded based on its related key capabilities. There are attempts to extend simple programming designs with cloud capabilities. In Cloud environment data is not stored on the user's computer but stored in the third parties cloud storage. The Cloud Computing infrastructure allows its resources to be accessed between users, servers and can openly access data/file stored in the cloud server. Due to this open access factor, one person's files or data is used by other users of the cloud, resulting in more or less dangerous to treat data or files. Once intruders have access to data, its abuse is a major threat. Intruder destroys the actual data or break off the communication. Cloud service providers provide critical security over files and data that require much attention to security. One of the most common problems in the cloud is that the person does not control the data storage space. Cloud Service Provider provides a cloud user provided resource allocation and scheduling facility, when processed when required to protect files/data. To defeat this problem, security in the cloud server should be effective. 


\section{DATA SECURITY USING ENCRYPTION}

Security is a key element in wide range of areas like network security, data security and user access mechanisms. In Cloud Computing one of the major area is data security, encryption mechanisms, data resiliency and replication, data availability and integrity are some of the popular methods to provide data security in cloud computing. In these methods we have used encryption method by using RSA and Modified RSA algorithms. In cloud-based data transmissions most commonly used protocol is HTTPS, which uses SSL/TLS underlying protocol. Most of the Transport Layer Security implementations used by RSA algorithm because it is the chief asymmetrical encryption cipher.

Generally Cloud Services has more harm than the intra network services and also additional security problems arise on virtualization and outsourced resources. The major Cloud Security problems are raised based on the selection of Cloud Service Model and runtime of the applications. To identify the security problems on the cloud environment we have to analyze the following steps:

1. Identify the resource: Identify which type of resource (Data/Applications or Services) to deploy on the cloud environment.

2. Identify the access level of the resource: Identify the risks that that are associated on the resource related to availability, privacy, unauthorized access and loss of data.

3. Identify the deployment model: Cloud deployment models are private, public, community and hybrid. Identify which type of deployment model is appropriate for your resource.

4. Determine the delivery model: Different cloud delivery models are available such as IaaS, PaaS and SaaS. Identify which type of delivery model is suitable for your IT resource.

5. Evaluation of Cloud Provider: Users need to assess the system to know where data is stored and how data is transferred in and out of the cloud environment after identify resources offered by a cloud provider.

Infrastructure is provided by IaaS, Frameworks for application development, manage structures and transactions provided by PaaS and an Operating Environment with applications, effective user interface and management provided by SaaS. From the existing service models IaaS has the least integrated functionality so it requires minimum security but SaaS has more integrated functionality so we require more security [4].

$\mathrm{SaaS}$ is delivery model for shared cloud services that can be located as commercialized products hosted by clouds. In this model cloud consumer is given access the cloud service contact, but not any underlying IT resources or implantation details. The PaaS cloud delivery model enables a cloud provider to offer a preconfigured environment that cloud consumers can use to build and deploy cloud services and solutions, even though with decreased administrative control. A Cloud Consumer is accessing a ready-made PaaS environment. IaaS cloud delivery model offers cloud consumers a high level administrative control over "raw" infrastructure-based IT resources. A cloud consumer is using a virtual server with in an IaaS environment. Cloud consumers are provided with range of contractual guarantees by the cloud provider, pertaining to characteristics such as capacity performance and availability.

\section{RSA ALGORITHM}

RSA authentication and the identity of service provider over insecure communication medium. Cracking the RSA encryption is at most as difficult as factoring huge numbers [5, 6]. The RSA algorithm involves three steps $[7,8]$ :

1. Generation of Key

2. Encryption by using Public Key and

3. Decryption by using Private Key.

Select two different prime numbers $\mathrm{p}$ and $\mathrm{q}$.

The integer's $\mathrm{p}$ and $\mathrm{q}$ should be generated at randomly because of security.

compute $\mathrm{n}=\mathrm{p} * \mathrm{q}$;

compute $f(n)=(p-1)(q-1)$

choose e such that e is relatively prime to $f(n)$ and less than $f(n)$.

find out $d$ such that de congruent modulo $1(\bmod f(n))$ and $d<f(n)$.

Public key $=\{e, n\}$, Private key $=\{d, n\}$

Cipher text $\mathrm{c}=$ message $\mathrm{e} \bmod \mathrm{n}$

Plain text $\mathrm{p}=$ ciphertext $\mathrm{d} \bmod \mathrm{n}$ 


\section{MODIFIED RSA MODEL}

Figure 1 show the encryption and decryption using MDRSA.

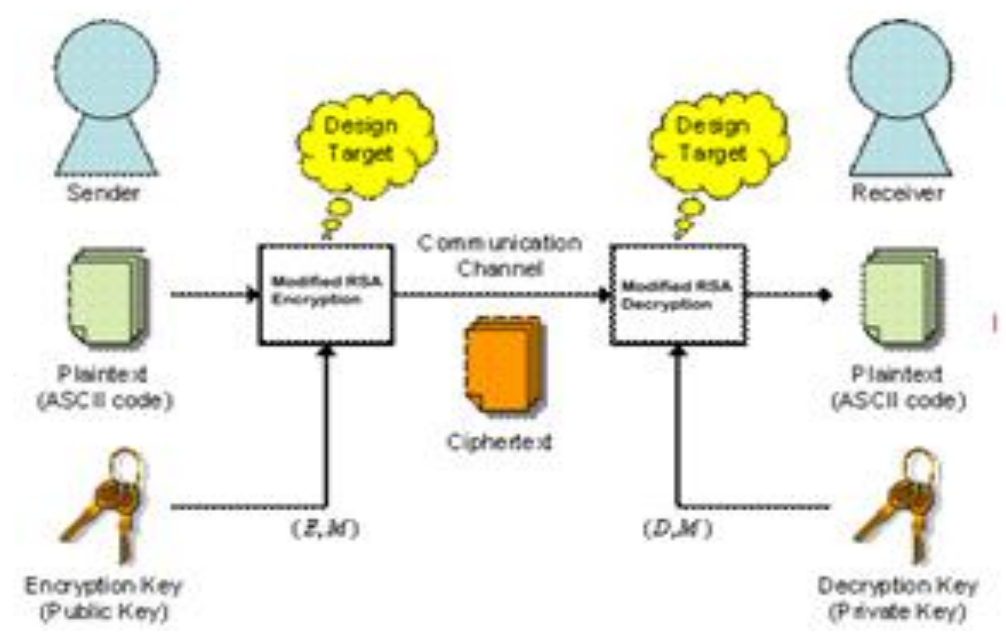

Figure 1. Encryption and decryption using MDRSA

We divide the program into 3 parts

1. plaintext + publickey1 --> ciphertext1

2. ciphertext $1+$ publickey $2-->$ ciphertext 2

3. ciphertext1 + private key --> plain text

In the majority cases the problem of RSA is breakable as of simply calculation of keys based on N, because it is the only product of two prime numbers, $\mathrm{N}$ can be easily noticeable. Once the value of $\mathrm{N}$ is recognized, an intruder can recover the keys and crack the system. The most important modifications which build the system is capable and secure are considered in the modified RSA Model [9, 10].

Modified RSA algorithm is performed by following way:

1. $\mathrm{n} 1=\mathrm{p} \times \mathrm{q}$

2. $\mathrm{n} 2=\mathrm{rx} \mathrm{s}$

3. $\mathrm{N}=\mathrm{n} 1 * \mathrm{n} 2$

4. $\mathrm{ph}=(\mathrm{p}-1)(\mathrm{q}-1)(\mathrm{r}-1)(\mathrm{s}-1)$

5. $\mathrm{e}=\mathrm{e} 1 * \mathrm{e} 2$

6. Calculate e 1 and e 2 and multiply two values (e $1 * \mathrm{e} 2)$ or calculate e and divide e into e 1 and e 2 then we get two encryption dan one decryption.

The modified RSA Model generates randomly four large prime numbers $\mathrm{p}, \mathrm{q}, \mathrm{r}$ and $\mathrm{s}$. There are three components consisting in public key generation e, $\mathrm{f}, \mathrm{N}$ where e and $\mathrm{f}$ are at random generated and also three components consisting in private key generation $\mathrm{d}, \mathrm{g}, \mathrm{N}$ where $\mathrm{d}$ and $\mathrm{g}$ are randomly generated $[11,12]$. In customized RSA model more complexity number is $\mathrm{N}$. In public and private key generation $\mathrm{N}$ is the same value, an intruder with the information of $\mathrm{N}$ cannot find out the values of four prime numbers which are essential for finding the value of $\mathrm{N}$ and consequently e and $\mathrm{f}[13,14]$.

\section{RESULTS AND DISCUSSIONS}

Key Size: [1024]

Generated prime numbers $\mathrm{p}, \mathrm{q}, \mathrm{r}$ and $\mathrm{s}$

$\mathrm{p}$ :

[DBB0B0485D335DF044F4EDD18415D5A43BAF2C9FB8A99BE5347E910138D19AF24788AF77801DE 68E47BBB85AA839DC374A539B3AE6F8449389BDC5FCEF3F6298C06C17264BA809FD7EC50FE0D9 A7753EF8F2C30216046------]

q:

[96D0AF060D3116D4686A5156CD44FF53B647376816B3F34D00BB7A0DAF048627A49494716E41DA 822D5734976DA6FB5EBA062CC913758E6208E93D6D7E4320331CE85C841E031095B0FDBA7730BD8 5F3CFD8FB9B1CAD15C------] 
r:

[8DD21B80761BBF6C8C0DB42F70CBB9DE0962DC0C19DE32D77830B6856BE09E5158C302C94F982 12982086BB09DF8098064F848B8D4401D544E37C76D0B1C7033B431037FEAD1557296626188B20DF1 756E2B7A7191B2C72D4FB7A----]

s:

[9FEBECA150EB7E87E6F63C8E20E19F8112379CA8BF0FA7F474EB0BC6662BE29C2CD50B22330EB 0CA0D6BC9FE3288A275A706845CC518C989F2FBC1FD3001C18FBAE3BFA3505912B72D411A5DCD CA18A941255699432494C1D2162-----]

Public Key pair $n$ and $\mathrm{E}$

Generated Value of N:

[2CCA47C6672AA889B2FBB67F1D71B6C4884A8CB7B0EBBD5FFEB52507C83DA918A509C858A389 37CDC68DDB55C20FE7F44C9195048F5E00447C66734D9094BDC164F067C68AD7470FF784282FBD3 2E123309C469E360EDF9-----]

Generated Value of E:

[D12E253C6B9A2D1C4DAF5890FB3D5ACBB8CBE7736E37AC70B3AEFEEC5AEF2B2AC2484C9E769 AF1D44831A9DDE0EF2E767269FFD07F99854B4A0583EAF4457B7907B2F50EBC55C4A34656E4AA6 2190477ECEF5BA8FF8CD0D01DB-----]

Private Key pair $\mathrm{n}$ and $\mathrm{D}$

Generated Value of N:

[2CCA47C6672AA889B2FBB67F1D71B6C4884A8CB7B0EBBD5FFEB52507C83DA918A509C858A389 37CDC68DDB55C20FE7F44C9195048F5E00447DA64677C28F24FD8069389D8B6CFC66734D9094BDC 164F067C68AD7470FF784

Generated Value of D:

[1A9A8F639F9776D55E9C0C79E00728BDE804E7A9C513613E1C5197FD519E78A030719A6705439235 97D602D5F1A9F8DA1192D491A73B68D94F47F27396FD95135BFD88EA9EB1A17A11D776A3D471D5 F6394632FA100BA7D4AFE883A3-----]

Please enter message (plaintext):

This is secret information

Generated Cipher text:

[532076F90468DA03C7696D357210A2DDB35444D25D764A1D705535B320F25029DB786E7DF8114C4 C4D877A271318D41823AA1B7647DACB1E2A9B9A866F951F740056732D758DF177FA41B280D3764B F6D5B3202242210A9BF1E667-------]

Execution time for encryption in milliseconds: 3087.0078

Generated Cipher text1:

[1FAA52CC64E4A85AA9CD083C1116BC9BF07D90A8FD19A7C12C8C7FBEA0E976F788D5BA532F20 46D6D873E8031672A8A1A8B02772AB7F12679A647E1288417CF45B9E4AE6AE65D8DB49B6A6E3A3 C77E2B07B714E8BE2C56C7AF5-----]

Execution time for decryption in milliseconds: 3087.0078

Recovered plaintext: [This is secret information] Algorithm.

Figure 2 show time consumption for different key sizes by key generation using RSA and MDRSA

\begin{tabular}{c|c|c|c|c|}
\hline \multirow{2}{*}{$\begin{array}{l}\text { Key Size } \\
\text { (bits) }\end{array}$} & \multicolumn{2}{|c|}{ RSA (ms) } & \multicolumn{2}{c|}{ MDRSA (ms) } \\
\cline { 2 - 5 } & $\begin{array}{c}\text { Encryption } \\
\text { time }\end{array}$ & $\begin{array}{c}\text { Decryption } \\
\text { time }\end{array}$ & $\begin{array}{c}\text { Encryption } \\
\text { time }\end{array}$ & $\begin{array}{c}\text { Decryption } \\
\text { time }\end{array}$ \\
\hline 128 & 8.388608 & 5.24288 & 12.582912 & 12.582912 \\
\hline 256 & 14.68006 & 12.582912 & 74.4489 & 68.15744 \\
\hline 512 & 134.2177 & 134.2133 & 536.8709 & 536.8709 \\
\hline 1024 & 536.8709 & 402.6532 & 3087.0078 & 3087.0078 \\
\hline 2048 & 3489.661 & 3355.443 & 26172.457 & 26575.11 \\
\hline
\end{tabular}

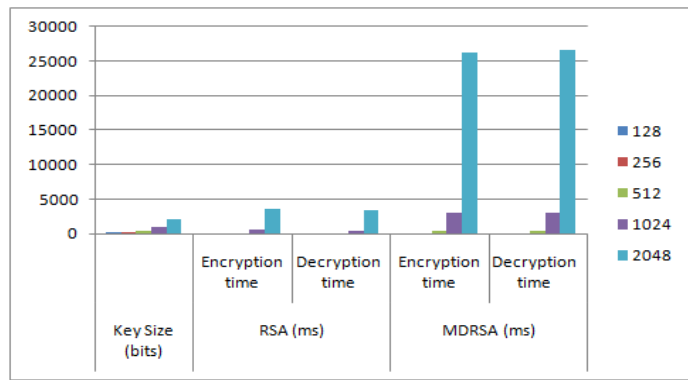

Figure 2. Time consumption for different key sizes by key generation using RSA and MDRSA Algorithm 
The RSA and Modified RSA models represent the encryption and decryption time in milliseconds based on key sizes from 128 bits to 2048 bits. We recommended that Modified RSA with key size 1024 bits will be a optimize solution which make balance between speed and security from the reaming key size values $[15,16]$.

\section{PERFORMANCE EVALUATION}

The proposed Modified RSA (MDSA) algorithm is examined on varying bit sizes of input. Performance of original RSA algorithm by Rivest, Shamir, and Adleman [17, 18] are shown in Table 1. Also the performance of Modified RSA (MDSA) scheme in terms of encryption time and decryption is shown in Table 2. Comparing the above tables, it can be concluded that the time of key generation of Modified RSA (MDSA) is higher than that of RSA. The higher key generation time of Modified RSA (MDSA) can be seen as an advantage by the fact that the time to break the system is high because of the extra complexity added. Figure 3 shown the encryption time comparison between RSA and proposed Modified RSA (MDSA) scheme [19, 20]. It illustrates that, for the lower bit length of prime numbers, two algorithms consume the almost identical amount of time. But with the increase of bit length, the difference between curves rises rapidly $[21,22]$.

Table 1. Encryption time comparison using RSA

\begin{tabular}{ccc}
\multicolumn{3}{c}{ and MDRSA Algorithms } \\
\hline $\begin{array}{c}\text { Key Size } \\
\text { (in bits) }\end{array}$ & $\begin{array}{c}\text { Encryption time in } \\
\text { ms (RSA) }\end{array}$ & $\begin{array}{c}\text { Encryption time } \\
\text { in ms (MDRSA) }\end{array}$ \\
\hline 128 & 0.19 & 0.68 \\
256 & 0.36 & 1.48 \\
512 & 0.57 & 3.2 \\
1024 & 1.72 & 7.8 \\
2048 & 3.33 & 21.92 \\
4096 & 11.18 & 56.88 \\
\hline
\end{tabular}

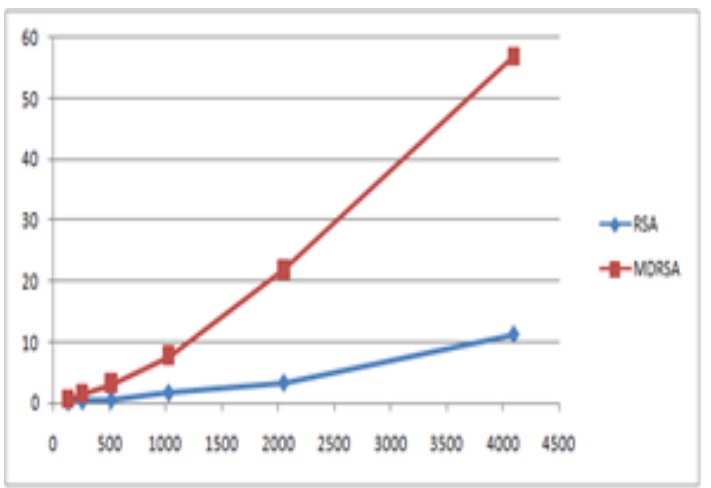

Table 2. Decryption time comparison using RSA and MDRSA Algorithms

\begin{tabular}{ccc}
\hline $\begin{array}{c}\text { Key Size } \\
\text { (in bits) }\end{array}$ & $\begin{array}{c}\text { Decryption time } \\
\text { in ms (RSA) }\end{array}$ & $\begin{array}{c}\text { Decryption time in } \\
\text { ms (MDRSA) }\end{array}$ \\
\hline 128 & 0.29 & 2.9 \\
256 & 0.98 & 14.28 \\
512 & 5.3 & 87.96 \\
1024 & 26.19 & 446.34 \\
2048 & 130.84 & 2472.72 \\
4096 & 1116.26 & 19983.38 \\
\hline
\end{tabular}

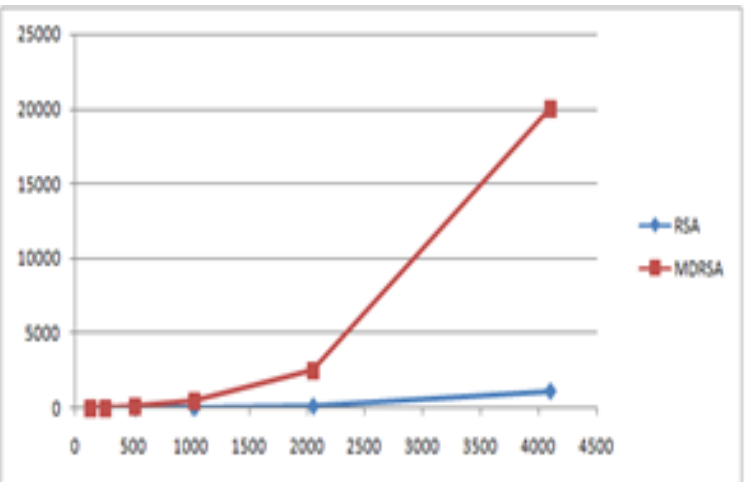

Figure 3. Encryption and decryption time comparison using RSA and MDRSA Algorithms based on key size

Figure 3 illustrate the encryption and decryption time comparison between RSA and proposed Modified RSA (MDSA) scheme. We recommended that Modified RSA with key size 1024 bits will be a optimize solution which make balance between speed and security from the reaming key size values $[23,24]$. With the increase of bit length, the difference between curves elevates rapidly at key size 2048 and 4096, it can be easily seen that encryption and decryption times are higher than RSA. The increase in time is adaptable because it increases the security to a great extent in the proposed Modified RSA (MRSA) method [25].

\section{CONCLUSION}

This manuscript has proposed a structure to provide confidentiality protection to the data stored in cloud environment. The modified RSA scheme used to protect the data in such a way that no reveal of data on cloud. Thus, in our projected work, only the approved user can access the data using the private key can be correctly decrypted. If some unauthorized users gets the data by chance or intentionally if he/she captures 
the data also, he/she can't decrypt it and get back the original data from it. With the help of this new security model which is modified RSA Public Key Cryptosystem, we can improve the security flaw of existing data security model in cloud environment and thereby guarantee the data security in cloud environment. In this modified RSA algorithm verification is more secure than RSA algorithm.

\section{REFERENCES}

[1] Sosinsky, "Understanding Cloud Security," Cloud Computing Bible Sosinsky/Cloud, 2011.

[2] B. A. Forouzan, "Cryptography and Network Security," Tata McGraw Hill Education Private Limited, New York, 2010.

[3] M. B. Mollah, et al., "Next generation of computing through cloud computing technology,"2012 25th IEEE Canadian Conference on Electrical and Computer Engineering (CCECE), 2012.

[4] Thomas E., "Cloud Computing, Concepts, Technology \& Architecture," Pearson Publication,2014.

[5] M. A. Islam, et al., "A Modified and Secured RSA Public Key Cryptosystem Based on "n" Prime Numbers,"Journal of Computer and Communications, 2018.

[6] I. G. Amalarethinam and H. M. Leena, "Enhanced RSA Algorithm with Varying Key Sizes for Data Security in Cloud,"2017 World Congress on Computing and Communication Technologies (WCCCT), Tiruchirappalli, pp. 172-175, 2017.

[7] Y. Pachipala, et al., "Data Security in Cloud using RSA," 2013 Fourth International Conference on Computing Communications and Networking Technologies (ICCCNT), 2013.

[8] P. Garg and V. Sharma. "An efficient and secure data storage in Mobile Cloud Computing through RSA and Hash function,"International Conference on Issues and Challenges in Intelligent Computing Techniques (ICICT), 2014.

[9] A.V.N. Krishna, "Chapter 16 A Randamized Cloud Library Security Environment,” IGI Global,2014.

[10] R. Biswas, et al., "A fast implementation of the RSA algorithm using the GNU MP library," IIIT - Calcutta.

[11] S. Sharma, "RSA algorithm using modified subset sum cryptosystem,"Computer and Communication Technology (ICCCT), pp. 457-461, 2011.

[12] A. Mousa, "Sensitivity of Changing the RSA Parameters on the Complexity and Performance of the Algorithm,"Journal of Applied Science,Asian Network for Scientific Information,vol. 5, pp. 60-63,2005.

[13] M. A. Islam, et al., "A Modified and Secured RSA Public Key Cryptosystem Based on "n" Prime Numbers,"Journal of Computer and Communications, vol. 6, pp. 78-90, 2018.

[14] M. Thangavel, et al., "An Enhanced and Secured RSA Key Generation Scheme (ESRKGS),"Journal of Information Security and Applications, 2015.

[15] R. S. Dhakar, et al.,"Modified RSA Encryption Algorithm (MREA),"2012 Second International Conference on Advanced Computing Communication Technologies, Rohtak, pp. 426-429, 2012.

[16] H. M. Sun, et al.,"Dual RSA and Its Security Analysis,"IEEE Transactions on Information Theory, vol. 53, pp. 2922-2933, 2007.

[17] T. C. Segar and R. Vijayaragavan, "Pell's RSA Key Generation and Its Security Analysis,"2013 Fourth International Conference on Computing, Communications and Networking Technologies (ICCCNT), Tiruchengode,pp. 1-5, 2013.

[18] M. J. Wiener, "Cryptanalysis of Short RSA Secret Exponents,"IEEE Transactions on Information Theory, vol. 36, pp. 553-558, 1990.

[19] A. H. Al-Hamami and I. A. Aldariseh, "Enhanced Method for RSA Cryptosystem Algorithm,"2012 International Conference on Advanced Computer Science Applications and Technologies (ACSAT),pp. 402-408, 2012.

[20] Y. Li, et al.,"Design and Implementation of an Improved RSA Algorithm,"2010 International Conference on E Health Networking Digital Ecosy stems and Technologies ( EDT ), Shenzhen, vol. 1, pp. 390-393, 2010.

[21] A. Chhabra and S. Mathur, "Modified RSA Algorithm: A Secure Approach,"2011 International Conference on Computational Intelligence and Communication Networks, Gwalior, pp. 545-548, 2011.

[22] B. Swami, et al.,"Dual Modulus RSA Based on Jordan-totient Function, Procedia Technology, vol. 24, pp. 15811586, 2016.

[23] R. Patidar and R. Bhartiya, "Modified RSA Cryptosystem Based on Offline Storage and Prime Number,"2013 IEEE International Conference on Computational Intelligence and Computing Research, Enathi, pp.1-6, 2013.

[24] R. L. Rivest, et al., "A Method for Obtaining Digital Signatures and Public-key Cryptosystems,"Communications of the ACM , vol. 21, pp. 120-126, 1978.

[25] K. Govinda, et al.,"Data Security in Cloud using Blowfish Algorithm,"International Journal for Scientific Research \& Development, vol. 2, 2014. 


\section{BIOGRAPHIES OF AUTHORS}

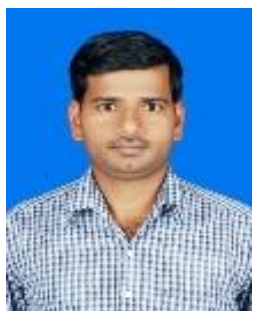

Mr. Y.Kiran Kumar, M.C.A, (Ph.D), he received his Master of Computer Applications from Sri Venkatesra University,Tirupati. He is Pursuing Ph.D from Bharathiar University, Coimbatore. He is having more than 10 years of teaching experience, Currently he is working as a Assistant Professor in the department of M.C.A in Sree Vidyanikethan Engineering College, Affiliated by JNTUA, Ananthapuramu, India. His areas of research interests include Web Technologies, Service Oriented Architecture and Cloud Computing.

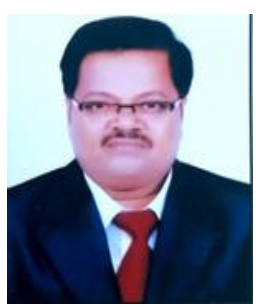

Dr. R. Mahammad Shafi, M.C.A, M.Tech, Ph.D He received his Ph.D from University of Allahabad, Allahabad. He is having more than 20 years of teaching experience. His areas of research interests include Software Engineering, Software Testing and Quality Assurance. He has published papers in refereed journals and conference proceedings in these areas. He has been involved in conferences and workshops as a Committee member, organizer and Session Chair. 